\title{
Three-dimensional SiGe/Si heterostructures: Switching the dislocation sign by substrate under-etching
}

\author{
Fabrizio Rovaris, ${ }^{1}$ Fabio Isa, ${ }^{2,3}$ Riccardo Gatti, ${ }^{4}$ Arik Jung, ${ }^{2,3}$ Giovanni Isella, ${ }^{5}$ Francesco Montalenti,,${ }^{1, *}$ and Hans von Känel ${ }^{2,3}$ \\ ${ }^{1}$ L-NESS and Department of Materials Science, University of Milano-Bicocca, Via R. Cozzi 55, I-20125 Milan, Italy \\ ${ }^{2}$ Laboratory for Solid State Physics, ETH Zürich, Otto-Stern-Weg 1, 8093, Zürich, Switzerland \\ ${ }^{3}$ Electron Microscopy Center Empa, Swiss Federal Laboratories for Materials Science and Technology, \\ Überlandstrasse 129, 8600, Dübendorf, Switzerland \\ ${ }^{4}$ Laboratoire d'Etude des Microstructures, UMR 104 CNRS-ONERA, 29 Avenue de La Division Leclerc, F-92322 Chatillôn, France \\ ${ }^{5}$ L-NESS, IFN-CNR and Dipartimento di Fisica, Politecnico di Milano, Via Anzani 42, Como I-22100, Italy
}

(Received 29 September 2017; published 22 December 2017)

\begin{abstract}
We present a joint theoretical and experimental analysis of the dislocation distribution in graded epitaxial $\mathrm{SiGe}$ crystals grown on under-etched Si pillars by low-energy plasma-enhanced chemical vapor deposition. Dislocation dynamics simulations are used to investigate preferential positioning of $60^{\circ}$ dislocations introduced in the system to release the lattice misfit strain. Coupling to a finite-element solver is exploited to allow for the exact numerical treatment of the stress fields in the presence of a complex distribution of free surfaces. The results show that, by suitably under-etching the Si pillars, it is possible to reverse the sign of the Burgers vector of the dislocations. This helps explaining differences in the experimentally observed distribution of dislocations in SiGe crystals grown on vertical and under-etched pillars, leading to a strong reduction of defects in the latter case. The agreement between simulations and experiments is not simply qualitative: the predicted number of defects generated by multiplication processes in tall crystals is indeed fully consistent with the measured one.
\end{abstract}

DOI: 10.1103/PhysRevMaterials.1.073602

\section{INTRODUCTION}

The epitaxial growth of lattice-mismatched semiconductors on silicon has attracted widespread attention as it offers the possibility to exploit superior optical and transport properties of the deposited materials while maintaining compatibility with the mainstream Si technology. This was demonstrated not only for SiGe material investigated in this work [1-3], but also for III/V semiconductors, such as GaAs [4], GaN [5], GeSn [6], and others. In most of the applications, a thin film (from a few $\mathrm{nm}$ to some $\mu \mathrm{m}$, depending on the targeted application) is deposited on a flat $\mathrm{Si}$ wafer, such as $\mathrm{Si}(001)$. Due to the lattice mismatch, elastic energy is accumulated in the film up to a certain critical thickness, beyond which plastic relaxation sets in [7]. In some cases, the insertion of dislocations can be preceded by the formation of three-dimensional (3D) islands [8], partially relieving the stress. However, this only postpones the unavoidable formation of linear defects $[9,10]$ at sufficient material coverages. Since islanding increases the film roughness, suitable deposition procedures freezing the adatom surface-diffusion lengths and hindering their formation have been devised [11-13]. Islanding can also be avoided by slowly grading the lattice mismatch, i.e., by growing alloy films where the lattice mismatch is progressively increased during the deposition. For example, pure Ge films on silicon can be grown by first depositing $\mathrm{Si}_{1-x} \mathrm{Ge}_{x}$ alloys, with linearly increasing Ge content $x$ with the film thickness. Such virtual substrates [14] proved to be extremely appealing in terms of reducing the threading-dislocation densities (TDDs) below $10^{7} \mathrm{~cm}^{-2}$. Nowadays, the development of suitable deposition/annealing/etching strategies has allowed to reach similarly

\footnotetext{
*francesco.montalenti@unimib.it
}

low values also for direct Ge/Si film deposition [15]. The remarkable reduction of the TDD is of particular importance since those defects have detrimental impacts on the properties of integrated devices [16]. However, continuous shrinking of the devices dimensions calls for a further decrease in the TDD, as even a single defect could be sufficient to severely alter the desired functionality. More complex techniques aimed at producing dislocation-free materials in the selected active areas have been developed. Those are aspect ratio trapping, in which the epitaxial material is selectively deposited into small windows of an oxide mask, and the TDs are geometrically confined close to the heterointerface [17-19], pendeoepitaxy (leading to the formation of a suspended film starting from a suitable seed layer [20]), and 3D heteroepitaxy [21-23]. In the latter, $\mathrm{Ge}$ (or $\mathrm{SiGe}$ ) is deposited onto an ordered array of micrometric, square Si pillars. The combined effect of the largely strong out-of-equilibrium growth conditions (high deposition rate of $\approx 5 \mathrm{~nm} / \mathrm{s}$, low growth temperatures $\leqslant 750{ }^{\circ} \mathrm{C}$ ), and mutual shadowing among neighboring pillars, leads to the formation of vertical heterostructures, whose upper region is fully dislocation free as defects are laterally expelled. By combining the 3D heteroepitaxy approach with the compositional grading, $100 \%$ dislocation-free, micronsized SiGe crystals were recently demonstrated [24]. The difference between grading in standard planar films and in vertical heterostructures is substantial. In the first case, the compositional grading only influences the distribution/density of dislocation threading arms, resulting in films with a TDD of $\approx 10^{5} \mathrm{~cm}^{-2}$ [14]. In the second, it allows to release completely the lattice-mismatch strain only by exploiting lateral elastic relaxation (similarly to what happens in nanowires [25], but at the micron scale [26]). As a result, neither misfit dislocations nor TDs are introduced. Vertical compositionally graded heterostructures, however, still need further optimization before 

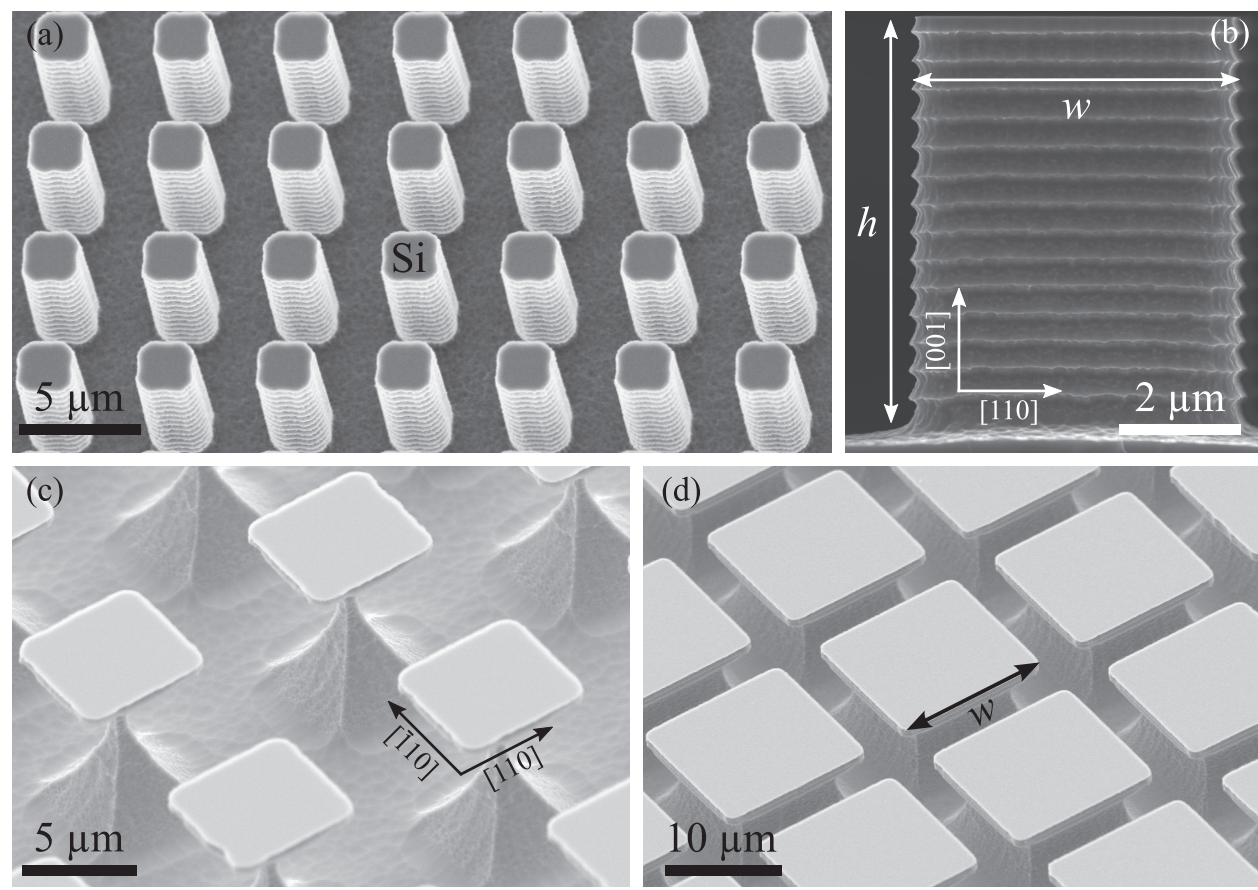

FIG. 1. Perspective SEM view of a Si pillars array with vertical $\{110\}$ sidewalls. (b) Cross-sectional SEM image of one Si pillar with vertical $\{110\}$ sidewalls, width $w=5 \mu \mathrm{m}$, and patterned height $h=7 \mu \mathrm{m}$. The scallops on the pillar sidewalls are typical for the Bosch etching process. (c), (d) Perspective view SEM images of under-etched Si pillars with $w=7$ and $15 \mu \mathrm{m}$, respectively.

being easily exploited in applications. Indeed, for a fixed final Ge content target $\left(x_{f}\right), 100 \%$ dislocation-free crystals can be obtained only by a proper choice of the crystal width and/or Ge compositional grading rate $R$ [26]. Narrow crystals and shallow compositional grading rate favor the elastic stress relaxation mechanism. In Ref. [24], $R$ was set to $0.015 \mu \mathrm{m}^{-1}$, requiring some $30 \mu \mathrm{m}$ of deposited material to achieve dislocation-free heterostructures with $x_{f}=0.4$ and a lateral size of $5 \mu \mathrm{m}$. Obtaining the same result for larger lateral dimensions would be possible in theory but unpractical in terms of deposition time because of the required even lower grading rate. A recent paper has, however, opened interesting perspectives. By changing the Si pillar shape via a suitable under-etching procedure [27], it was experimentally shown that the dislocation density can be further lowered (bringing it back down to zero, in some cases) even for larger crystal widths.

In this paper, we analyze in depth, theoretically and experimentally, the role Si pillar under-etching plays in influencing the behavior of dislocations. By using dislocation dynamics simulations and elasticity-theory calculations, we show that the sign of the dislocation Burgers vector can be actually reversed. This finding allows for a direct interpretation of the measured distributions of dislocations in SiGe crystals grown as reported in [27]. The calculations of the optimal number of dislocations in pileups lead to predictions verified by our experimental measurements. The excellent agreement allows us to draw an overall picture of strain relaxation in vertical heterostructures and provide us guidelines for suitably tuning the shape of the substrate pattern to reduce the defects density in epitaxial SiGe crystals.

\section{EXPERIMENTS: GROWTH/ETCHING AND CHARACTERIZATION}

The SiGe epitaxial growth was performed by low-energy plasma-enhanced chemical vapor deposition (LEPECVD) (Ref. [28]) on 4-in $(10-\mathrm{cm})$ patterned $\mathrm{Si}(001)$ wafers. The $\operatorname{Si}(001)$ wafers were patterned in two different ways [Figs. 1(a), 1(b) and 1(c), 1(d), respectively]. Both types of patterns are based on optical lithography and reactive ion etching. The first one [see Figs. 1(a) and 1(b)] consists of fabrication by the Bosch process [29] of regular arrays of square, vertical Si pillars with a height $h$ of $8 \mu \mathrm{m}$. Further details about substrate patterning and preparation are reported in Ref. [23]. The resulting pillar width $w$ ranges from 2 to $50 \mu \mathrm{m}$, and the sides are aligned with the $\langle 110\rangle$ crystal directions. The second type [see Figs. 1(c) and 1(d)] involves two different dry etching steps. Initially, (0.6-1)- $\mu \mathrm{m}$ thick square mesas are produced by vertical etching, after which $3 \mu \mathrm{m}$ of isotropic etching is performed. This approach results in $\mathrm{Si}$ pillars consisting of a thin square mesa and an under-etched neck. The width of the pillar neck is determined by the width of the mesa (ranging from $w=7$ to $50 \mu \mathrm{m}$ ), assuming that the isotropic etching always removes $3 \mu \mathrm{m}$. For further details, see Ref. [27].

The epitaxial growth consists of three different steps. First, a 50-nm thin $\mathrm{Si}_{1-x} \mathrm{Ge}_{x}$ layer with $x_{l}=0.5 \%$ is deposited at $750{ }^{\circ} \mathrm{C}$ and $5.9 \mathrm{~nm} / \mathrm{s}$. Second, the compositionally graded alloy is grown at the Ge grading rate of $1.5 \% \mu \mathrm{m}^{-1}$ up to the final Ge content $x_{f}=0.4$. The Ge content is increased stepwise by $\Delta x=0.5 \%$. The growth temperature is kept constant at $750{ }^{\circ} \mathrm{C}$ for $x \leqslant 0.1$, while for larger Ge content, it linearly decreases to the final value of $590^{\circ} \mathrm{C}$ reached 

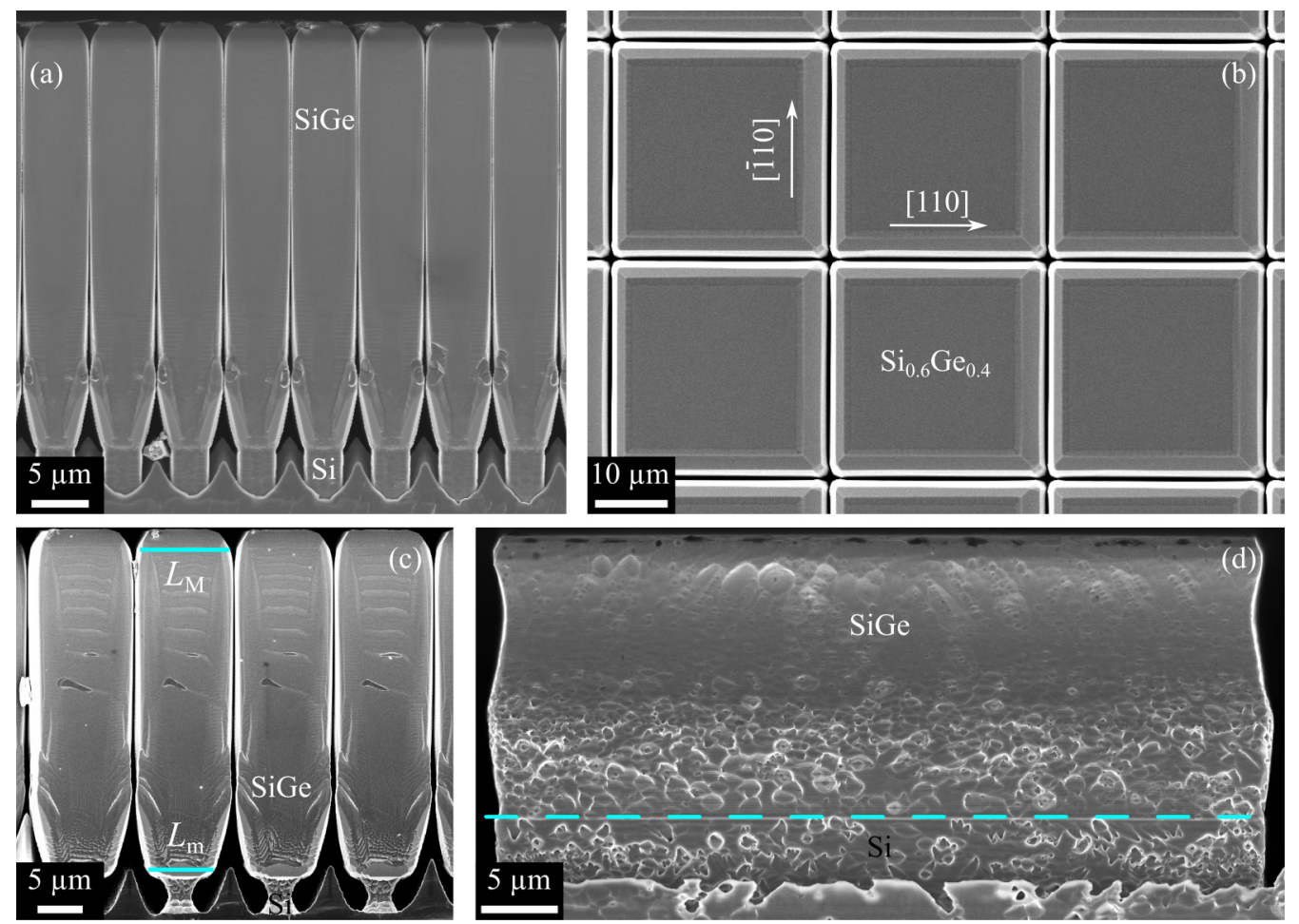

FIG. 2. (a), (b) Cross-sectional and top-view SEM images of SiGe crystals deposited on vertical Si pillars with $w=2$ and $25 \mu \mathrm{m}$, respectively. (c) The same of (a) but for under-etched Si pillars with $w=7 \mu \mathrm{m}$. Here, the two light blue lines indicate the extreme values of the SiGe crystal width taken at the SiGe/Si interface $\left(L_{\mathrm{m}}\right)$ and at the crystal top $\left(L_{\mathrm{M}}\right)$. (d) Cross-sectional SEM image after selective defect etching of a SiGe/Si crystal. Emerging dislocations, both in the SiGe crystal and in the Si pillar, appear as whitish pits. The dashed light blue line marks the $\mathrm{SiGe} / \mathrm{Si}$ heterointerface.

at $x_{f}=0.4$. Third, a $1-\mu \mathrm{m}$ thick capping layer at $x_{f}=$ 0.4 is deposited at $590^{\circ} \mathrm{C}$ and $6.2 \mathrm{~nm} / \mathrm{s}$. As illustrated in Figs. 2(a)-2(c), the epitaxial depositions result in several micrometers tall, isolated SiGe crystals for all the investigated pillar dimensions. The SiGe crystals are constituted by $\{110\}$ sidewalls and on top a central (001) surface bounded by $\{113\}$ facets [30].

The dislocation analysis is performed first of all by cleaving the samples along the [110] crystal directions, then by carrying out selective defect etching by a Cr-based solution, and finally by extensive SEM imaging to count etch pits related to dislocations (Ref. [31]). The dislocation density (DD) is calculated by taking into account a total $\{110\}$ sidewall surface of at least an equivalent area of $1.0 \times 10^{4} \mu \mathrm{m}^{2}$, corresponding to at least 20 pillars to ensure a reliable statistics. Figure 2(d) shows a typical example of the etch pits found on the $\{110\}$ crystal sidewalls. Dislocations are present both in the $\mathrm{SiGe}$ crystal and in the Si pillar, the former above and the latter below the dashed light blue line. We denote the corresponding two distinct dislocation densities in the $\mathrm{SiGe}$ crystal and the $\mathrm{Si}$ pillar as $\mathrm{DD}_{\mathrm{SiGe}}$ and $\mathrm{DD}_{\mathrm{Si}}$, respectively. In a recent publication [27], we demonstrated that Si pillar under-etching drastically reduces the $\mathrm{DD}_{\mathrm{SiGe}}$ compared to that observed for vertical $\mathrm{Si}$ pillars. In this section, we shall experimentally quantify this effect allowing for a detailed comparison with the simulations reported in Sec. IV.

The experimentally determined distribution of dislocations within the $\mathrm{SiGe/Si}$ crystals is elucidated in Fig. 3. Figure 3(a) shows the average $\mathrm{DD}_{\mathrm{SiGe}}$ as a function of the width of the
Si pillars and their geometry (vertical or under-etched). As already reported in Refs. [24,31], narrow and vertical Si pillars allow for a strong elastic relaxation of the epitaxial strain, preventing the nucleation of dislocations. Indeed, the $\mathrm{DD}_{\mathrm{SiGe}}$ (black spheres) is 0 for $2-\mu \mathrm{m}$ wide Si pillars, while it reaches a value of $\approx 4 \times 10^{7} \mathrm{~cm}^{-2}$ for $w=50 \mu \mathrm{m}$. In under-etched Si pillars, a much lower $\mathrm{DD}_{\mathrm{SiGe}}$ is measured in a wide range of $\mathrm{Si}$ pillar widths $w$ (red triangles) [27]. The additional compliance effect provided by under-etching becomes less and less effective with increasing $w$, and practically vanishes for $w=50 \mu \mathrm{m}$. This is due to the fact that the under-etching is constantly $3 \mu \mathrm{m}$ for every pillar width, while the Si neck becomes wider proportionally to $w$.

A lower $\mathrm{DD}_{\mathrm{SiGe}}$ is directly related to a higher probability of finding dislocation-free (DFP) SiGe crystals, named $\mathrm{DFP}_{\mathrm{SiGe}}$. This is explicitly shown in Fig. 3(b) in which we compare the $\mathrm{DFP}_{\mathrm{SiGe}}$ for SiGe crystals deposited on vertical (black spheres) and under-etched (red triangles) Si pillars of different widths. Apart from the SiGe crystals deposited on the narrowest $\mathrm{Si}$ pillars $(w=2 \mu \mathrm{m})$, which are $100 \%$ dislocation free, the $\mathrm{DFP}_{\mathrm{SiGe}}$ is higher for under-etched Si pillars.

On the contrary, Figs. 3(c) and 3(d) indicate that the density of dislocations located in silicon $\left(\mathrm{DD}_{\mathrm{Si}}\right)$ is larger in the case of under-etched pillars as compared to the case of vertical $\mathrm{Si}$ pillars. Figure 3(c) shows that the $\mathrm{DD}_{\mathrm{Si}}$ in vertical Si pillars increases with their width, being 0 for $w=2 \mu \mathrm{m}$, since in this case all the strain is elastically released $\left(\mathrm{DD}_{\mathrm{SiGe}}=0\right.$ as well), and reaching a value of $\approx 3 \times 10^{7} \mathrm{~cm}^{-2}$ for $w=$ $50 \mu \mathrm{m}$. Surprisingly, the $\mathrm{DD}_{\mathrm{Si}}$ of under-etched $\mathrm{Si}$ pillars 

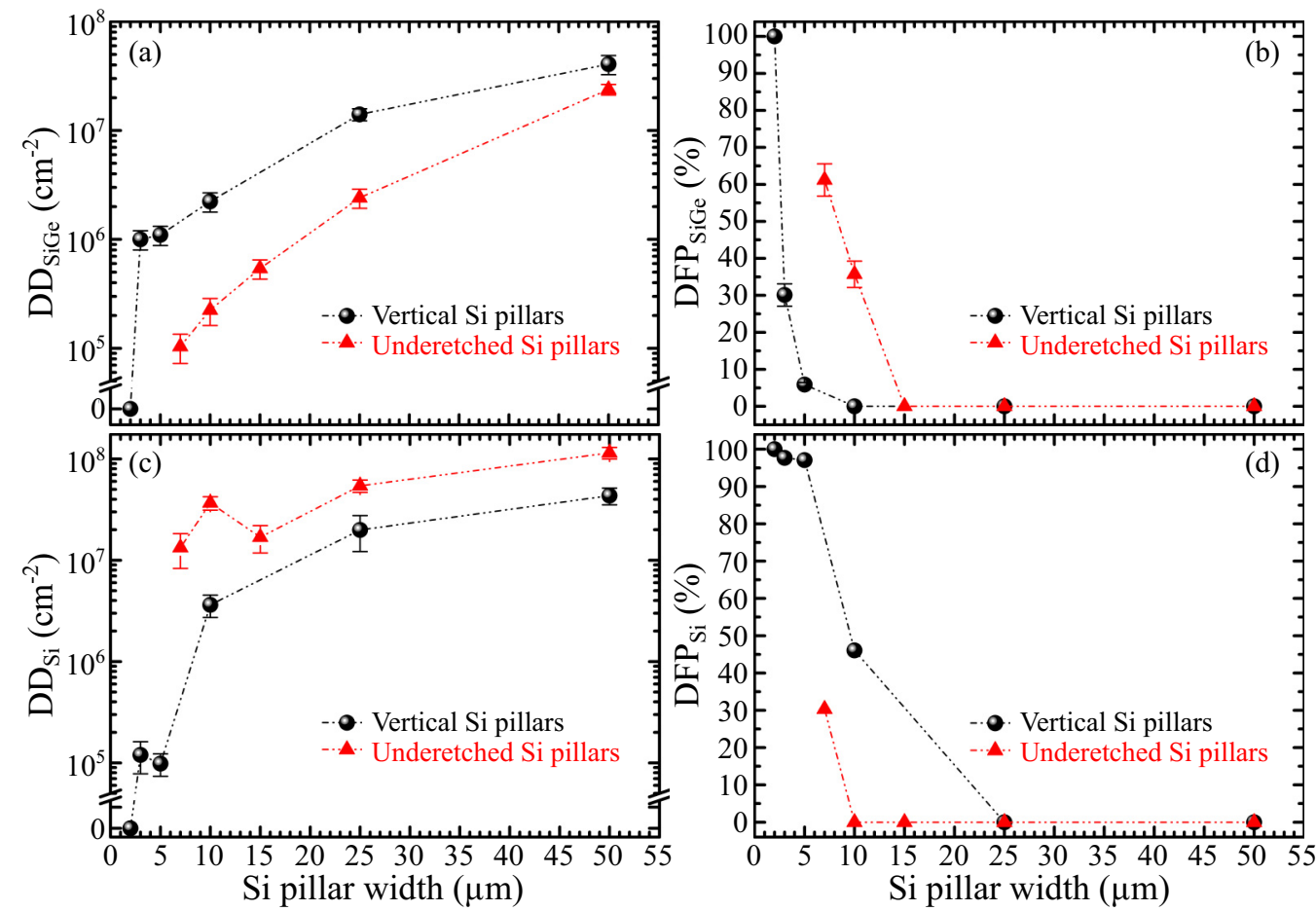

FIG. 3. (a) Average dislocation density in SiGe crystals $\left(\mathrm{DD}_{\mathrm{SiGe}}\right)$ deposited on vertical (black spheres) and under-etched (red triangles) Si pillars with different widths. (b) Probability of having dislocation-free $\mathrm{SiGe}$ crystals $\left(\mathrm{DFP}_{\mathrm{SiGe}}\right)$ as a function of the vertical (black spheres) and under-etched (red triangles) Si pillars width. (c), (d) Analogous to (a) and (b), respectively, but for dislocations located in the Si pillars.

(red triangles) is higher for every Si pillar width, increasing from $\approx 1 \cdot 10^{7} \mathrm{~cm}^{-2}$ to $1 \times 10^{8} \mathrm{~cm}^{-2}$ between $w=7$ and $50 \mu \mathrm{m}$. Analogously, Fig. 3(d) shows that the dislocation-free probability of under-etched Si pillars (red triangles) is much lower than that of vertical Si pillars (black spheres). The DFP ${ }_{S i}$ of under-etched Si pillars is only 30\% for $w=7 \mu \mathrm{m}$ and it drops to 0 for larger widths. On the other hand, the $\mathrm{DFP}_{\mathrm{Si}}$ of vertical Si pillars is nearly $100 \%$ for $w \leqslant 5 \mu \mathrm{m}$ and more than $45 \%$ for $w=10 \mu \mathrm{m}$. It only reaches 0 for pillars wider than $25 \mu \mathrm{m}$. A quantitative modeling of this phenomenon is given in Sec. IV, however, from the experimental results reported in Fig. 3, it is already clear that for small Si pillar widths, where elastic strain relaxation plays a fundamental role, the dislocation dynamics is strongly influenced by the Si pillar geometry. Indeed, for narrow Si pillars $(w \leqslant 10 \mu \mathrm{m})$ the $\mathrm{DD}_{\mathrm{SiGe}}$ is much lower and the $\mathrm{DFP}_{\text {SiGe }}$ much higher for deposition on under-etched Si pillars, while the $\mathrm{DD}_{\mathrm{Si}}$ and the $\mathrm{DFP}_{\mathrm{Si}}$ behave in the opposite way. This is not the case for wide $\mathrm{Si}$ pillars (i.e., $w=50 \mu \mathrm{m}$ ) where $\mathrm{DD}_{\mathrm{SiGe}}$ and $\mathrm{DD}_{\mathrm{Si}}$ are not geometry dependent and the $\mathrm{DFP}_{\mathrm{SiGe}}$ and $\mathrm{DFP}_{\mathrm{Si}}$ are both 0 .

In order to understand the different dislocation behavior, we take a closer look at the spatial distribution of dislocations within the narrow SiGe crystals. Figures 4(a) and 4(b) show cross-sectional SEM images of SiGe/Si crystals deposited on narrow vertical and under-etched Si pillars, respectively. The dashed light blue line indicates the $\mathrm{SiGe} / \mathrm{Si}$ heterointerface. In Fig. 4(a) the thickness of the layer with $x_{1}=0.5 \%$ is $8 \mu \mathrm{m}$. This ensures vertical growth of the compositionally graded material by reducing the gap between adjacent crystals, without altering the mechanical properties of the system. It is evident that the etch pits related to emerging dislocations are distributed very differently in the two panels. Importantly, dislocations are predominantly located in the SiGe crystal for vertical Si pillars [white arrow in Fig. 4(a)], while they are predominantly located in the Si region in the case of und-eretched pillars and piled up along the same $\{111\}$ glide plane [indicated by the red dashed ellipses in Fig. 4(b)]. Indeed the measured angle in Fig. 4(b) between the [110] direction and the dislocation pileup indicated by the orange arrows is about $54^{\circ}$, as expected from the intersection between the $\{111\}$ glide planes of the dislocations and the [110] direction. By looking at dozens of different under-etched Si pillars, as those of Fig. 4(b), it is possible to measure the probability of having a certain number of dislocations per pileup. Figure 4(c) shows the probability distribution of dislocations per pileup in under-etched $\mathrm{Si}$ pillars indicating that three dislocations per pileup is the most likely.

Let us now provide a theoretical explanation for the observed decrease (increase) of the number of dislocations in SiGe crystals (underlying Si pillar) as caused by under-etching.

\section{DISLOCATION DYNAMICS SIMULATIONS}

\section{A. Method}

The approach exploited in this paper to model the plastic relaxation provided by dislocations is a two-dimensional (2D) coupling between a dislocation dynamics code [32] and a finite-element (FE) solver, implemented in MATLAB [10], in the isotropic linear elasticity framework. The coupling is based on the eigenstrain formalism, reported in Ref. [33], numerically 


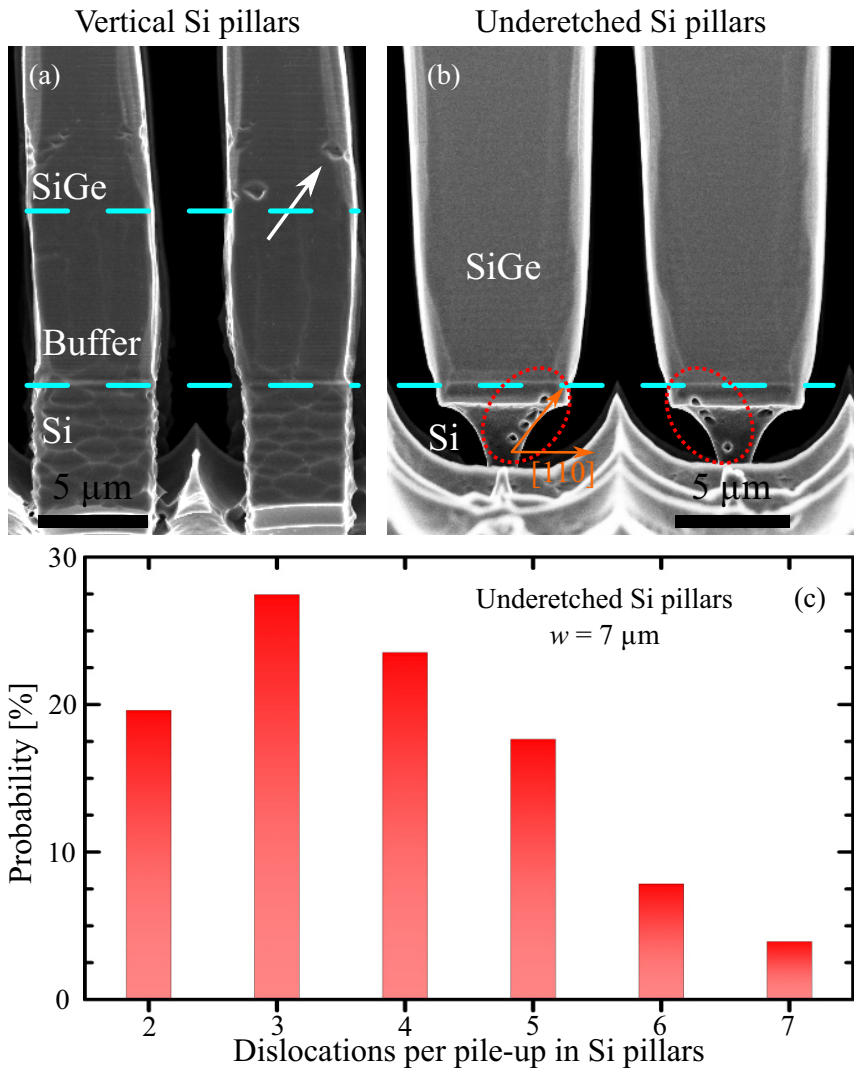

FIG. 4. (a), (b) Cross-sectional SEM images of SiGe crystals deposited on vertical and under-etched Si pillars, respectively, after selective defect etching (width $w=5$ and $7 \mu \mathrm{m}$ ), respectively. The dashed light blue line shows the $\mathrm{SiGe} / \mathrm{Si}$ heterointerface. The white arrow indicates a dislocation in the SiGe crystal. The red dashed ellipses in (b) highlight dislocations piled up along $\{111\}$ in the $\mathrm{Si}$ pillars. (c) Probability distribution of having a certain number of dislocations per pileup along $\{111\}$ planes in under-etched Si pillars $(w=7 \mu \mathrm{m})$.

implemented following the discrete-continuous model (DCM) scheme described in Ref. [34]. In the DCM scheme, the plastic deformation produced by a dislocation in a crystal lattice during the dynamics in the dislocation dynamics code is spread over a finite area by a distribution function with a characteristic thickness $d$. In particular, the distribution function used in Ref. [34] and in this work is the one proposed by Cai et al. in Ref. [35] to develop a nonsingular theory of dislocations. In dislocation dynamics codes, the movement of dislocations follows the Peach-Koehler force reported in Eq. (1):

$$
F=(\sigma \cdot b) \times \xi,
$$

where $\sigma$ is the applied stress field at the position of the dislocation, $\boldsymbol{b}$ its Burgers vector, and $\boldsymbol{\xi}$ the dislocation line direction. In our coupling, to compute the applied stress field at the $n$th time step of the dislocation dynamics code, the plastic deformation produced by the movement of all the dislocations is imposed as an eigenstrain to the FE mesh. Similarly, the misfit strain arising from the lattice mismatch between the graded $\mathrm{Si}_{1-x} \mathrm{Ge}_{x}$ and the $\mathrm{Si}$ pillar underneath is modeled with an eigenstrain function linearly dependent on the vertical position. Then, the stress field in the simulated domain can be recovered solving the elastic problem by means of the FE solver, fully taking into account the complex boundary conditions of the free surfaces. The stress field resulting from the FE solver is plugged into Eq. (1) and used to compute the forces to move the dislocation at the $n$th +1 time step, following an iterative process. It is important to highlight that while the FE solver is in charge of computing the mechanical equilibrium, the dislocation dynamics code not only handles the movement of dislocations, but it manages also the potential reactions between dislocations, correctly modeling the plastic deformation of the simulated system. While in the dislocation dynamics approach the only information needed to compute the dynamics are the forces acting on the dislocations, our choice of the distribution function allows us also for the evaluation of the total elastic energy of the system by direct numerical integration of the (regularized) stress field.

\section{B. Onset of plasticity}

In order to understand the distribution of dislocations revealed in the experiments and how this is affected by underetching the Si pillar, we started by investigating the plasticity onset, i.e., the introduction of the very first dislocation in the system during growth. This was done by evaluating the total elastic energy and by taking the energy difference between the system with and without the dislocation as a function of the pillar height $H$. The onset of plasticity is found when introducing a dislocation brings the total energy to a net reduction, corresponding to negative values of $\Delta E$.

In these calculations, dislocations are placed at the $\mathrm{SiGe} / \mathrm{Si}$ interface, probing different lateral positions, for each different $H$, in order to find the minimum-energy position. The difference between the energy with the dislocation placed at that position and the defect-free system is called $\Delta E_{\min }$. The evaluations are done by considering the geometry of a pillar grown on an under-etched Si pattern of base $7 \mu \mathrm{m}$. As clearly visible in Fig. 2(c), the experimentally grown pillars show a degree of taper in the vertical directions resulting from a certain degree of lateral growth. In our model, we considered a simplified geometry with constant pillar width (Fig. 5) for both vertical and under-etched Si pillars. In order to take care of the dependence of the onset of plasticity with the pillar width, we repeated the calculation for the two limiting values of width measured at the $\mathrm{SiGe} / \mathrm{Si}$ interface $\left(L_{m}=6.6 \mu \mathrm{m}\right)$ and at the pillar top $\left(L_{M}=11 \mu \mathrm{m}\right)$ [see also Fig. 2(c)], maintaining a constant neck for the under-etched region of $2 \mu \mathrm{m}$. We started by considering the $60^{\circ}$ dislocation Burgers vectors $b=[101] a_{\mathrm{Si}} / 2$ or $b=[101] a_{\mathrm{Si}} / 2$ (where $a_{\mathrm{Si}}$ is the lattice parameter of $\mathrm{Si}$ ) which features a compressive lobe mainly positioned in the silicon substrate and a tensile lobe in the SiGe epilayer. Such dislocations are typically encountered in $\mathrm{SiGe} / \mathrm{Si}$ heteroepitaxy of standard 2D films and also in vertical pillars grown with the same parameters of the samples considered in this paper (see Refs. [24,26]) and are introduced to relax the compressive strain in the $\mathrm{SiGe}$ epilayer. However, contrary to what happens in standard vertical pillars, the introduction of a dislocation with one of these two Burgers vectors inside an under-etched pillar is never energetically favored, as shown in the red region of Fig. 6 . 
(a)

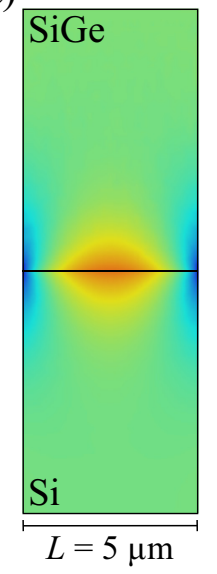

(b)

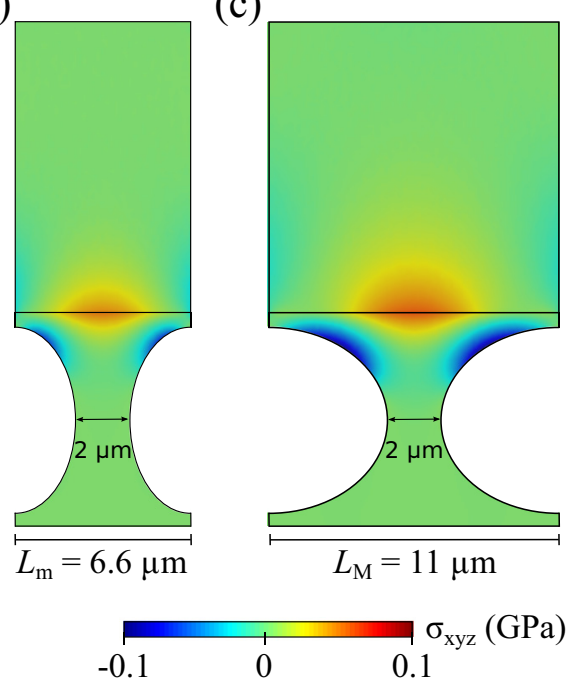

(d)

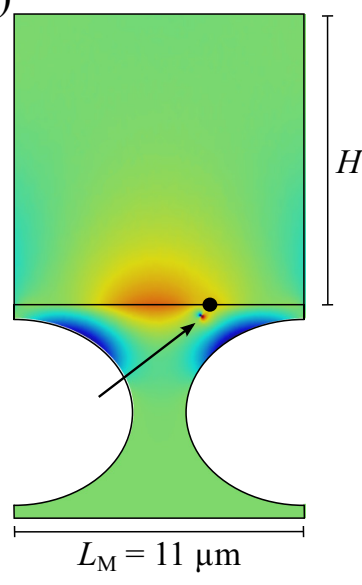

FIG. 5. Hydrostatic stress maps $\left(\sigma_{x y z}\right)$ and considered geometry for the vertical (a) and under-etched (b), (c) Si pillars. The SiGe/Si interface is marked with a black line. The two considered pillar bases $L_{\mathrm{m}}$ (b) and $L_{\mathrm{M}}$ (c) for the under-etched pillar are taken to mimic the extreme values of bases measured on the tapered geometry of the grown pillar as reported in Fig. 2(c). In (d) is reported the stress map for an under-etched pillar with the first dislocation introduced. The filled black circle reports its optimal position along the interface while the black arrow represents the result of the energy minimization by means of a dislocation dynamics simulation.

Surprisingly, the introduction of dislocations with the opposite sign of the Burgers vector (and opposite orientation for the compressive/tensile lobes) becomes energetically favorable after a certain critical thickness for both the considered values of pillar base (purple region in Fig. 6). In order to understand this evidence, it is convenient to look at the stress field reported in Figs. 5(b) and 5(c). As it is evident by comparing with Fig. 5(a), in the presence of under-etching a strongly compressed region is created in the underneath Si pillar close to the free surfaces of the neck. In order to release such stress, it is required to introduce a dislocation which adds space in silicon, i.e., the opposite of what happens in the vertical geometry or in standard $\mathrm{SiGe} / \mathrm{Si}$ heteroepitaxial films.

As we already emphasized, the values displayed in Fig. 6 were obtained, for simplicity, by placing the dislocation at the $\mathrm{SiGe} / \mathrm{Si}$ interface, in the horizontal position minimizing

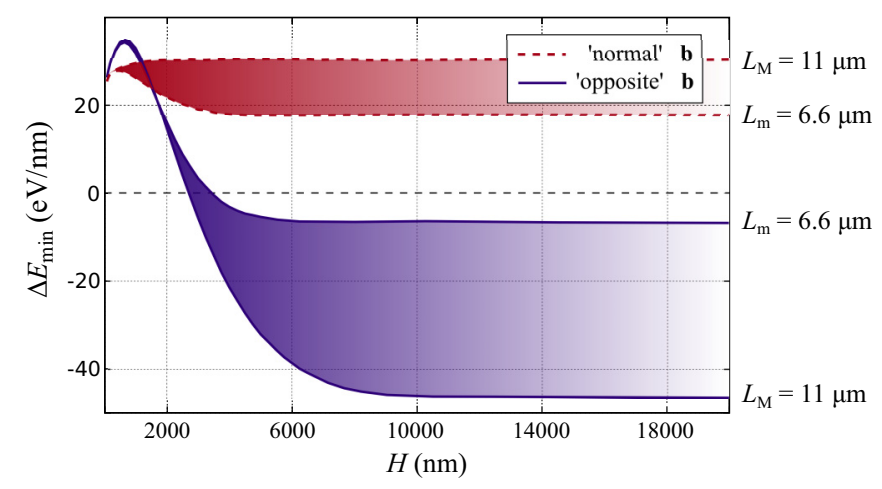

FIG. 6. Energy gain for the introduction of the first dislocations in an under-etched pillar with base $L$ with respect to the pillar height $H$. The formation energy is negative only for dislocations with the opposite Burgers vector $b$. the energy. Such a position is indicated, for $L_{\mathrm{M}}=H=$ $11 \mu \mathrm{m}$, by a black filled circle in Fig. 5(d). By running dislocation dynamics simulations starting from such a position we found out that a slightly lower-energy position located under the interface exists [tip of the black arrow in Fig. 5(d)]. However, the energy difference between the two produces negligible changes in terms of the estimate of the critical thickness.

The above thermodynamic approach (i.e., purely based on an energy minimization criterion), used to estimate the onset of plasticity, cannot be reliably extended to treat more advanced stages of relaxation (e.g., by looking at the minimum energy position for a larger number of dislocations) as nucleation processes are known to dominate in low-misfit graded layers $[7,36]$. The experimental images of Figs. 4(a) (vertical) and 4(b) (under-etched) are therefore exploited to proceed with further modeling. They show that dislocations tend to pile up on the same glide plane, a clear sign of multiple nucleation from the same source. Under this hypothesis, we tried to model the observed distribution of dislocations exploiting the dislocation dynamics approach described in Sec. III A. Results are discussed and compared with experiments in the next section.

\section{COMPARISON BETWEEN THEORY AND SIMULATIONS}

In order to predict the dislocation distribution, under the hypothesis of dislocation nucleation from the same source, we have placed a first dislocation at the $\mathrm{SiGe} / \mathrm{Si}$ interface, with both the Burgers vector and the lateral positioning derived in the previous section for both vertical and under-etched $\mathrm{Si}$ pillars. Then, an iterative procedure is performed by adding another identical dislocation to the same glide plane 


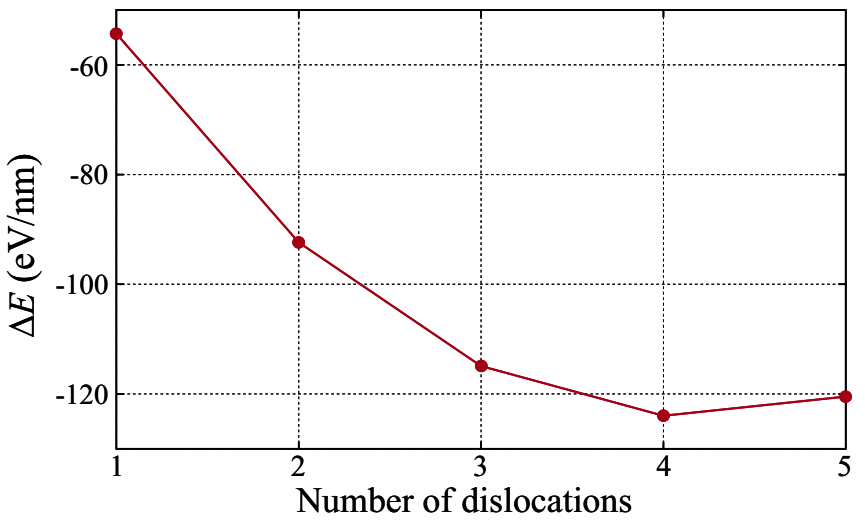

FIG. 7. Plot of the energy gain versus the number of dislocations per pileup in an under-etched pillar. The optimal number of dislocations can be deduced to be four by the minimum in this curve because the introduction of a further one will increase the total elastic energy.

and finding the resulting stable configuration by means of dislocation dynamics simulations. In the simulations, the first dislocation is kept fixed to the initial position as it is treated as the origin of the source (possibly originated in the 3D system by crossing between dislocations running in perpendicular direction and leading to multiplication [37]). Iterations are repeated until the total energy of the system is lowered by the addition of a further dislocation. Results are shown in Fig. 7 where the energy gain is plotted against the number of dislocations per pileup. As we can see, the minimum of this curve corresponds to a number of four dislocations, fully compatible with the experimental results of Fig. 4(c).

At the end of these simulations we are able to predict the optimal position of a pileup inside a pillar along with the total number and the positions of the individual dislocations inside the pileup. The final results of these computations are shown in Figs. 8(b)-8(d), while experimental SEM images are reported in Figs. 8(b)-8(c). In Figs. 8(a)-8(b), we can see how in under-etched pillars dislocations are pushed inside the silicon region underneath, and this is well comparable to the simulation results. In Figs. 8(c)-8(d), representing a standard vertical pillar, instead, the pileups tend to float in the SiGe region, again showing a behavior well comparable with the experimental results. Some differences in the theoretical vs experimental distributions can be spotted. This is not surprising taking into account some simplifications considered in the model where the system is described only in $2 \mathrm{D}$ and the first dislocation is kept fixed at the exact minimum energy position.

Finally, we wish to comment about another difference in the defects of vertical vs under-etched pillars, revealed by the SEM images of Figs. 8(a)-8(c). We recall that misfit and threading dislocations can be distinguished by the etch pits symmetry [31]. Indeed, misfit dislocations with line along the $\langle 110\rangle$ directions generate fourfold-symmetric etch pits, while the inclined threading dislocation line results in elongated and asymmetric etch pits. It is clearly visible in Figs. 8(a) and $8(\mathrm{c})$ that the etch pits in the vertical pillars are the result of a threading arm extending toward the lateral surface of the crystal, while in the under-etched ones they result from misfit segments running all across the pillar. We ascribe this difference to the different positioning of the dislocations. In the
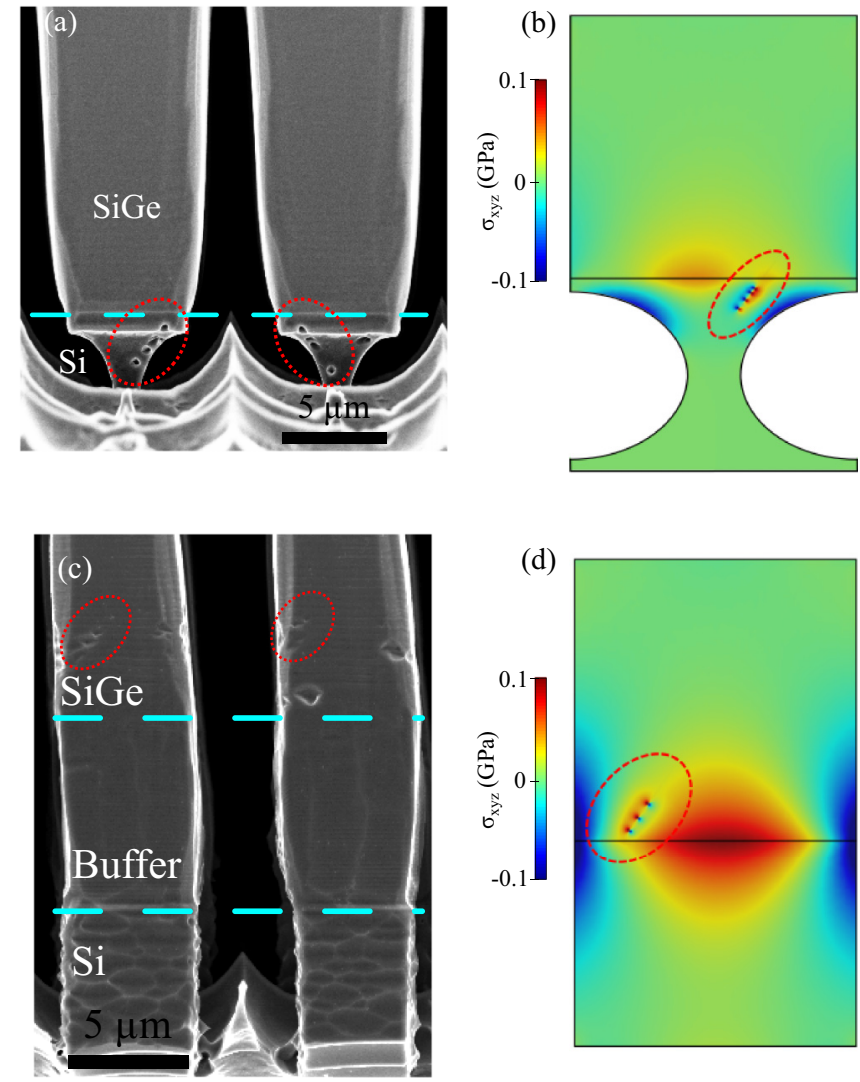

FIG. 8. Comparison between experimental SEM images and hydrostatic stress maps from the final stages of the dislocations dynamics simulations for an under-etched pillar [(a) and (b)] and a vertical one [(c) and (d)]. Experimental images in (a) and (c) are the same as in Fig. 4 and reported here for convenience. As we can see, the simulation can well reproduce the behavior shown in experiments with dislocations in vertical pillars floating in the $\mathrm{SiGe}$ region, while those in the under-etched ones are pushed into the silicon pillar underneath.

under-etched case, multiplication takes place closer to the free surfaces. Therefore, dislocation (semi)loops can more easily fully open, threading arms being expelled in the process.

\section{CONCLUSIONS}

In this work, we have presented a combined theoretical and experimental investigation of dislocation distributions in graded $\mathrm{SiGe}$ crystals grown on vertical and under-etched $\mathrm{Si}$ pillars. We have shown that the under-etching plays a dominant role by effectively inverting the sign of dislocation Burgers vectors. The modeling of the typical dislocation positioning as caused by multiplication leads to nice agreement with experiments both in terms of distribution and in the number of defects. We expect this study to trigger further research, e.g., on optimal shaping of the pillars, with the aim of growing larger dislocation-free SiGe crystals.

\section{ACKNOWLEDGMENTS}

F. Rovaris and F. Montalenti acknowledge financial support from Regione Lombardia, under the TEINVEIN project, Call Accordi per la Ricerca e l'Innovazione, co-funded by POR 
FESR 2014-2020 (Project ID No. 242092). F. Isa, A. Jung, and H. von Känel acknowledge the Sinergia Project NOVIPIX No. CRSII2_147639 of the Swiss National Science Foundation.
We are grateful to P. Niedermann from the CSEM, A. Ballabio and the technical staff at the L-NESS laboratory in Como, and FIRST clean room at the ETH Zürich.
[1] H.-D. Y. Kang, M. Liu, M. Morse, M. Paniccia, S. Zadka, G. Litski, A. Sarid, Y.-H. Pauchard, H.-W. Kuo, W. Chen et al., Nat. Photonics 3, 59 (2009).

[2] Y. Tokuda and E. Yagyu, Nat. Photonics 3, 7 (2009).

[3] R. Soref, Nat. Photonics 4, 495 (2010).

[4] W. Wang, Appl. Phys. Lett. 44, 1149 (1984).

[5] S. Guha and N. Bojarczuk, Appl. Phys. Lett. 72, 415 (1998).

[6] S. Wirths, R. Geiger, N. von den Driesch, G. Mussler, T. Stoica, S. Mantl, Z. Ikonic, M. Luysberg, S. Chiussi, J. Hartmann et al., Nat. Photonics 9, 88 (2015).

[7] R. Hull, in Properties of Silicon Germanium and SiGe: Carbon, edited by E. Kasper and K. Lyutovich (Inspec, Exeter, UK, 2000), pp. 21-41.

[8] D. Eaglesham and M. Cerullo, Phys. Rev. Lett. 64, 1943 (1990).

[9] F. K. LeGoues, M. C. Reuter, J. Tersoff, M. Hammar, and R. M. Tromp, Phys. Rev. Lett. 73, 300 (1994).

[10] F. Rovaris, R. Bergamaschini, and F. Montalenti, Phys. Rev. B 94, 205304 (2016).

[11] C. Rosenblad, E. Muller, and H. von Känel, Thin Solid Films 318, 11 (1998).

[12] L. Colace, G. Masini, F. Galluzzi, G. Assanto, G. Capellini, L. D. Gaspare, E. Palange, and F. Evangelisti, Appl. Phys. Lett. 72, 3175 (1998).

[13] A. Sakai, N. Taoka, O. Nakatsuka, S. Zaima, and Y. Yasuda, Appl. Phys. Lett. 86, 221916 (2005).

[14] E. Fitzgerald, Y.-H. Xie, M. Green, D. Brasen, A. Kortan, J. Michel, Y.-J. Mii, and B. Weir, Appl. Phys. Lett. 59, 811 (1991).

[15] Y. Yamamoto, G. Kozlowski, P. Zaumseil, and B. Tillack, Thin Solid Films 520, 3216 (2012).

[16] P. Taylor, W. Jesser, J. Benson, M. Martinka, J. Dinan, J. Bradshaw, M. Lara-Taysing, R. Leavitt, G. Simonis, W. Chang et al., J. Appl. Phys. 89, 4365 (2001).

[17] J.-S. Park, J. Bai, M. Curtin, B. Adekore, M. Carroll, and A. Lochtefeld, Appl. Phys. Lett. 90, 52113 (2007).

[18] J. Li, J. Bai, J.-S. Park, B. Adekore, K. Fox, M. Carroll, A. Lochtefeld, and Z. Shellenbarger, Appl. Phys. Lett. 91, 21114 (2007).

[19] J. Bai, J.-S. Park, Z. Cheng, M. Curtin, B. Adekore, M. Carroll, A. Lochtefeld, and M. Dudley, Appl. Phys. Lett. 90, 101902 (2007).

[20] K. Linthicum, T. Gehrke, D. Thomson, E. Carlson, P. Rajagopal, T. Smith, D. Batchelor, and R. Davis, Appl. Phys. Lett. 75, 196 (1999).
[21] A. Marzegalli, F. Isa, H. Groiss, E. Müller, C. V. Falub, A. Taboada, P. Niedermann, G. Isella, F. Schffler, F. Montalenti et al., Adv. Mater. 25, 4408 (2013).

[22] F. Isa, A. Marzegalli, A. Taboada, C. V. Falub, G. Isella, F. Montalenti, H. von Känel, and L. Miglio, APL Mater. 1, 52109 (2013).

[23] C. V. Falub, H. von Känel, F. Isa, R. Bergamaschini, A. Marzegalli, D. Chrastina, G. Isella, E. Muller, P. Niedermann, and L. Miglio, Science 335, 1330 (2012).

[24] F. Isa, M. Salvalaglio, Y. Dasilva, M. Meduňa, M. Barget, A. Jung, T. Kreiliger, G. Isella, R. Erni, F. Pezzoli et al., Adv. Mater. 28, 884 (2016).

[25] F. Glas, Phys. Rev. B 74, 121302 (2006).

[26] M. Salvalaglio and F. Montalenti, J. Appl. Phys. 116, 104306 (2014).

[27] F. Isa, M. Salvalaglio, Y. A. R. Dasilva, A. Jung, G. Isella, R. Erni, B. Timotijevic, P. Niedermann, P. Grning, F. Montalenti, and H. von Känel, Appl. Phys. Lett. 109, 182112 (2016).

[28] C. Rosenblad, H. R. Deller, A. Dommann, T. Meyer, P. Schroeter, and H. von Känel, J. Vac. Sci. Technol. A 16, 2785 (1998).

[29] F. Laermer and A. Schilp, Method of anisotropically etching silicon (1996), US Patent No. 5,501,893.

[30] R. Bergamaschini, F. Isa, C. Falub, P. Niedermann, E. Maeller, G. Isella, H. von Känel, and L. Miglio, Surf. Sci. Rep. 68, 390 (2013).

[31] F. Isa, M. Salvalaglio, Y. A. R. Dasilva, A. Jung, G. Isella, R. Erni, P. Niedermann, P. Gräning, F. Montalenti, and H. von Känel, Acta Mater. 114, 97 (2016).

[32] D. Gómez-García, B. Devincre, and L. P. Kubin, Phys. Rev. Lett. 96, 125503 (2006).

[33] T. Mura, in Mechanics of Elastic and Inelastic Solids, 2nd ed., edited by S. Nemat-Nasser and G. A. Oravas, Mechanics of Elastic and Inelastic Solids, Vol. 3 (Springer, Dordrecht, 1987).

[34] O. Jamond, R. Gatti, A. Roos, and B. Devincre, Int. J. Plas. 80, 19 (2006).

[35] W. Cai, A. Arsenlis, C. R. Weinberger, and V. V. Bulatov, J. Mech. Phys. Sol. 54, 561 (2006).

[36] F. K. LeGoues, J. A. Ott, K. Eberl, and S. S. Iyer, Appl. Phys. Lett. 61, 174 (1992).

[37] K. W. Schwarz, J. Appl. Phys. 85, 120 (1999). 